\title{
Factores asociados a complicaciones infecciosas posoperatorias en pacientes con urolitiasis
}

\section{Factors Associated with Postoperative Infectious Complications in Patients with Urolitiasis}

\author{
Javier Osorio-Manrique ${ }^{1}$ Jorge Andres Ramos-Castaneda ${ }^{20}$ Elsa Garcia-Landazabal ${ }^{1}$ \\ Maria Paula Molano-Garcia ${ }^{3}$ \\ ${ }^{1}$ Centro Especializado de Urología. Neiva, Colombia \\ 2 Universidad CES, Medellín, Colombia \\ 3 Universidad Pedagógica y Tecnológica de Colombia, Tunja, Colombia \\ Address for correspondence Jorge Andres Ramos-Castaneda, PhD, \\ Universidad CES, Medellín, Cl. 10a \#22 - 04 \\ (e-mail: jormos2806@gmail.com).
} Urol Colomb 2021;30:105-111.

\section{Resumen}

Palabras clave

- urolitisiasis

- infección

- procedimiento urológico

\section{Abstract}

Introducción Se encuentran múltiples tratamientos para la urolitiasis, los cuales dependen en gran medida del tamaño y la ubicación de la litiasis. Dentro de las opciones terapéuticas se encuentran la expulsión activa, utilización de ondas de choque, las ureterolitotomias, y procedimientos endoscópicos, todas con diferentes tasas de efectividad y riesgo de complicación infecciosa. El objetivo del estudio fue evaluar los factores asociados a la complicación infecciosa posterior al procedimiento urológico en pacientes con urolitiasis.

Métodos Estudio de casos y controles anidado a una cohorte de pacientes programados para procedimientos urológicos como tratamiento para la urolitiasis durante el periodo 2015 - 2019. Se consideraron casos, aquellos con complicación infecciosa posoperatoria dentro de los 30 días posteriores al procedimiento urológico. Los controles se seleccionaron de la cohorte inicial y fueron los pacientes que no presentaron complicación infecciosa.

Resultados En el periodo de estudio, fueron atendidos 350 pacientes con litiasis renal, con un total de 1258 cálculos. El número promedio de cálculos por paciente fue de 3,59; la prevalencia de litiasis bilateral fue del $57 \%$, siendo similar la cantidad de cálculos en el lado izquierdo (promedio $=1,4$ ) y en el derecho (promedio $=1,44$ ). La tasa de complicación infecciosa posquirúrgica fue del $4 \%(n=14)$ y se asoció con la presencia de al menos un cálculo mayor de 20 mm (OR 4,49 IC95\% 1,39-14,52).

Conclusión La tasa de complicación infecciosa posoperatoria en pacientes con urolitiasis y programados para procedimientos urológicos es baja. Una longitud del cálculo mayor de $20 \mathrm{~mm}$ es un factor asociado a la infección posoperatoria.

Introduction There are multiple treatments for urolithiasis, which largely depend on the size and location of the lithiasis. Among the therapeutic options are active expulsion, use of shock waves, urolithotomies, and endoscopic procedures, all with different rates of effectiveness and risk of infectious complications. The objective of the received

June 9, 2020

accepted

August 25, 2020

published online

February 4, 2021
DOI https://doi.org/

10.1055/s-0040-1721322.

ISSN $0120-789 X$

e ISSN 2027-0119.

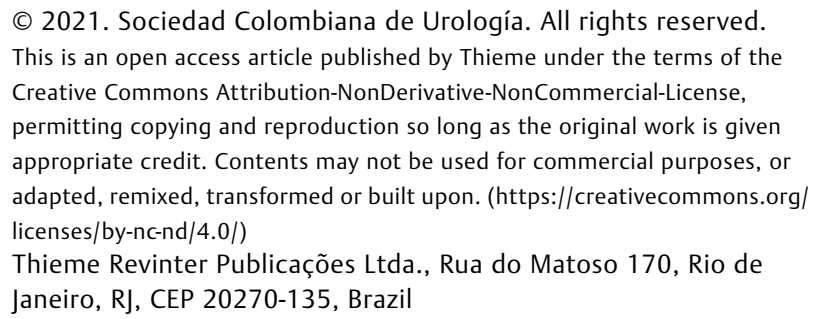

(C) 2021. Sociedad Colombiana de Urología. All rights reserved. This is an open access article published by Thieme under the terms of the Creative Commons Attribution-NonDerivative-NonCommercial-License, permitting copying and reproduction so long as the original work is given appropriate credit. Contents may not be used for commercial purposes, or adapted, remixed, transformed or built upon. (https://creativecommons.org/ licenses/by-nc-nd/4.0/)

Thieme Revinter Publicações Ltda., Rua do Matoso 170, Rio de Janeiro, RJ, CEP 20270-135, Brazil 
Keywords

- urolitisiasis

- infection

- urological procedure study was to evaluate the factors associated with the infectious complication after the urological procedure in patients with urolithiasis.

Methods Case-control study nested in a cohort of patients scheduled for urological procedures as treatment for urolithiasis during the period 2015-2019. Cases were considered, those with postoperative infectious complication within 30 days after the urological procedure. The controls were selected from the initial cohort and were the patients who did not present an infectious complication.

Results During the study period, 350 patients with kidney stones were treated, with a total of 1258 stones. The average number of stones per patient was 3.59; the prevalence of bilateral lithiasis was $57 \%$, the number of stones being similar on the left side (average $=1.4$ ) and on the right (average $=1.44$ ). The postoperative infectious complication rate was $4 \%(n=14)$ and was associated with the presence of at least one stone larger than $20 \mathrm{~mm}$ (OR 4.49 95\% Cl 1.39 - 14.52).

Conclusion The postoperative infectious complication rate in patients with urolithiasis and scheduled for urological procedures is low. A stone length greater than $20 \mathrm{~mm}$ is a factor associated with postoperative infection.

\section{Introducción}

La urolitiasis, también conocida como cálculos renales o litiasis renal, se refiere a la presencia de piedras (cálculos) en riñones, uréteres, vejiga o uretra, y se caracteriza por una variedad de síntomas como, dolor de alguno de los flancos, hematuria, náuseas y vómito., ${ }^{1,2}$ Se estima que dicho problema se presenta entre $2-3 \%$ de la población general, y en países industrializados puede llegar afectar entre el 5$13 \%$ de sus habitantes, ${ }^{6}$ siendo una patología con mayor predominio en hombres. ${ }^{6-8}$

La litiasis renal se encuentra relacionada con múltiples factores tales como, la edad, anomalías metabólicas (obesidad, diabetes mellitus, síndrome metabólico, gota), desnutrición, factores dietéticos, ingesta de líquidos, entre otros. ${ }^{6,9}$ Así mismo, la presencia de cálculos en el tracto urinario puede causar dolor lumbar, insuficiencia renal, sangrado en orina, cólico nefrítico, síndrome miccional irritativo, pionefrosis e infecciones. ${ }^{5,7}$ Por esa razón, se necesita de intervenciones y/o tratamientos que permitan liberar o eliminar los cálculos de los pacientes con el menor daño o complicaciones posibles.

En la literatura se encuentran múltiples tratamientos para la urolitiasis, los cuales dependen en gran medida del tamaño y la ubicación de la litiasis. ${ }^{10}$ Dentro de las opciones terapéuticas se encuentran la expulsión activa, utilización de ondas de choque, las ureterolitotomias, y procedimientos endoscópicos, todas con diferentes tasas de efectividad y riesgo de complicación $^{11}$. Se estima que la tasa de complicación en pacientes con nefrolitotomía percutánea (NLP) varía entre $11,9 \%$ y $27 \%$, siendo similar con la ureteroscopia flexible con tasas entre $6,25 \%$ y $29 \%^{5,12-14}$. Dentro de las complicaciones posteriores a procedimientos urológicos, se encuentran los sangrados (7,8\%), transfusión sanguínea $(5,7 \%)$, perforación de pelvis renal $(3,4 \%)$ e infección del tracto urinario, siendo esa última la más frecuente. ${ }^{5,10,15}$
Respecto a los factores asociados a complicaciones infecciosas posteriores al procedimiento urológico, la mayoría de los estudios se enfocan en comorbilidades, bacteriuria asintomática, catéter urinario, pero existe poca evidencia sobre la influencia de las características de los cálculos, tales como tamaño, cantidad, localización y bilateralidad. El objetivo del estudio, fue evaluar los factores asociados a la complicación infecciosa posterior al procedimiento urológico en pacientes con urolitiasis.

\section{Métodos}

\section{Tipo de estudio}

Se llevó a cabo un estudio de casos y controles basado en una cohorte de pacientes con litiasis renal y programados para procedimientos urológicos. La investigación se desarrolló en un centro especializado de urología en una ciudad de Colombia. La institución atiende en promedio a 5000 pacientes de urología por año.

\section{Población y muestra}

La cohorte fue de naturaleza retrospectiva ya que estuvo conformada por los pacientes con diagnóstico de urolitiasis y que tuvieron un procedimiento urológico como tratamiento en el periodo 2015 - 2019. En la institución donde se realizó el estudio, existe un comité de complicaciones quirúrgicas que realiza seguimiento telefónico hasta 30 días después del procedimiento a todos los pacientes intervenidos.

La cohorte estuvo conformada por 350 pacientes que tuvieron un procedimiento urológico como tratamiento de la litiasis renal. La selección de las unidades de información se realizó a través de un muestreo aleatorio simple. A partir de la cohorte se conformaron los grupos de casos y controles. Se consideraron casos aquellos que presentaron complicación infecciosa posoperatoria dentro de los 30 días posteriores al procedimiento urológico. Los controles se seleccionaron de la 
cohorte inicial y fueron los pacientes que no presentaron complicación infecciosa.

Se excluyeron las personas que fueron intervenidas quirúrgicamente en otra institución, pacientes con signos de infección previo al procedimiento urológico y los menores de 18 años.

\section{Variables analizadas}

Para el estudio se consideró la variable dependiente como la presencia de cualquier complicación infecciosa posoperatoria ocurrida durante los 30 días posteriores al procedimiento urológico. Dentro de la complicación infecciosa se consideró la infección de la herida quirúrgica (superficial, profunda y órgano espacio), infección del tracto urinario y sepsis. La infección fue captada mediante el reingreso a urgencia a la institución y al seguimiento telefónico que se realiza a todos los pacientes por parte del comité de complicaciones quirúrgicas.

Como variables independientes se analizaron las características de los cálculos, tales como, cantidad, ubicación, tamaño, densidad y bilateralidad. También se tuvieron en cuenta otras variables como sexo, edad, y antecedente quirúrgico 3 meses previos al procedimiento.

\section{Procedimientos e instrumentos}

La recolección de la información se realizó mediante revisión documental, que incluyó: historia clínica, bases de datos, reportes quirúrgicos y paraclínicos de los pacientes. Además del seguimiento telefónico que se les realiza a todos los pacientes intervenidos por parte del comité de complicaciones quirúrgicas.

\section{Sesgos}

Para evitar el sesgo de información diferencial, los datos fueron recolectados por los investigadores con previo entrenamiento y capacitación sobre el instrumento de recolección. También se contó con la supervisión de los investigadores principales durante el proceso de recolección de la información.

El estudio podría tener un sesgo de centro de referencia, ya que se trabajó con pacientes de una institución de salud, por lo que no se podrían generalizar los resultados a toda la población.

El sesgo de información se controló ya que la información no dependió de una entrevista al paciente, sino que se revisó la historia clínica. La información de los cálculos se tomó de los estudios de imagenología realizados en el paciente.

\section{Análisis estadístico}

Se realizó la comparación de los casos y controles en sus condiciones basales. Las variables categóricas fueron analizadas mediante proporción; las numéricas se comprobó su comportamiento normal mediante la prueba estadística Kolmogorov Smirnov. Aquellas que no presentaron un comportamiento normal, se analizaron con pruebas no paramétricas basadas en la mediana y su rango.

Para el análisis bivariado se utilizó la prueba de Chi cuadrado o test de Fisher (cuando se encontraron valores esperados menores a cinco) para variables categóricas y para las numéricas la prueba t Student (verificando la igualdad o diferencia de varianzas mediante la prueba Levene) o la prueba U de Mann - Whitney según la normalidad de la variable.

Se llevó a cabo análisis por regresión logística binaria donde la variable dependiente fue la complicación infecciosa. Como variables independientes se incluyeron las que en el análisis bivariado tuvieron una $\mathrm{p}<0,25$, aquellas con importancia clínica y las posibles confusoras. El modelo fue evaluado mediante una prueba de bondad de ajuste y con el coeficiente de determinación. Los resultados fueron expresados en Odds Ratio (OR) con sus intervalos de confianza del 95\%. Para el análisis estadístico se utilizó el programa R 3.3.3. ${ }^{16}$

\section{Declaración sobre aspectos éticos}

El estudio se clasificó sin riesgo según la resolución 8430/ 1993 y fue aprobado por el comité de la institución hospitalaria. No se recolectaron datos de información personal de los pacientes y todos los investigadores firmaron un acuerdo de confidencialidad con la institución.

\section{Resultados}

En el periodo de estudio fueron atendidos 350 pacientes con litiasis renal, con un total de 1258 cálculos. El promedio de la edad de los pacientes fue de 46,54 años y la mayoría fueron del sexo femenino ( $n=201,57 \%$ ).

El número promedio de cálculos por paciente fue de 3,59; la prevalencia de litiasis bilateral fue del $57 \%$, siendo similar la cantidad de cálculos en el lado izquierdo (promedio $=1,4$ ) y en el derecho (promedio $=1,44)(-$ Tabla 1 ). Se observó que cuatro de cada cinco pacientes tuvieron litiasis múltiple, y uno de cada tres tuvo un cálculo mayor a $10 \mathrm{~mm}$; el promedio de tamaño y densidad fue de $8,04 \mathrm{~mm}$ y 700,68 UH respectivamente ( $\mathbf{- T a b l a} \mathbf{1}$ ).

Los cálculos en la región del cálice se ubicaron principalmente en la zona inferior y media $(70,51 \%)$, mientras que los del uréter se localizaron en la región distal $(53,27 \%)$ (-Tabla 2).

Se observó diferencia significativa en el tamaño y la densidad de los cálculos según su ubicación. Las piedras ubicadas en la vejiga y la pelvis fueron más grandes que los que se encontraban en el cálice y en el uréter (- Figura 1). En la pelvis y la vejiga fue donde se encontraron las piedras de mayor y menor densidad, respectivamente.

La tasa de complicación infecciosa posquirúrgica fue del $4 \%(\mathrm{n}=14)$ y se asoció con una longitud promedio mayor de $6 \mathrm{~mm}$ (OR 5,93 IC95\% 1,31-26,90), y la presencia de al menos un cálculo mayor de $20 \mathrm{~mm}$ (OR 3,69 IC95\% 1,18-11,51) (-Tabla 3). La localización, la edad y el sexo no presentaron diferencias significativas en los pacientes con complicación infecciosa.

Los pacientes con complicaciones infecciosas posoperatorias tuvieron menor cantidad de cálculos en la lateralidad derecha, y se debe a que presentaron un mayor número de piedras con un tamaño menor de $6 \mathrm{~mm}$ (promedio 1,64 ), comparado con el lado izquierdo (promedio 1,46 ). 
Tabla 1 Variables clínicas de la litiasis de los pacientes con litiasis renal

\begin{tabular}{|l|l|}
\hline VARIABLES & $\begin{array}{l}\text { Total } \\
\text { N }=350\end{array}$ \\
\hline TOTAL, CAL & \\
\hline Media (D.E.) & $3,59(2,64)$ \\
\hline IZQ. CAL (número) & \\
\hline Media (D.E.) & $1,4(1,23)$ \\
\hline DER. CAL (número) & \\
\hline Media (D.E.) & $1,44(1,29)$ \\
\hline TAMAÑO (mm) & \\
\hline Media (D.E.) & $8,04(5,98)$ \\
\hline DENSIDAD TOTAL (UH) & \\
\hline Media (D.E.) & $700,68(395,32)$ \\
\hline MÚLTIPLES CÁLCULOS & \\
\hline Sí & $281(80 \%)$ \\
\hline No & $69(20 \%)$ \\
\hline BILATERAL & \\
\hline Sí & $198(57 \%)$ \\
\hline No & $152(43 \%)$ \\
\hline LOCALIZACIÓN & \\
\hline Cálice & $293(84 \%)$ \\
\hline Uréter & $185(53 \%)$ \\
\hline Pelvis renal & $32(9 \%)$ \\
\hline Uretra & $1(0,3 \%)$ \\
\hline Vejiga & $9(3 \%)$ \\
\hline TAMAÑO & \\
\hline Mayor o igual 10 mm & $116(33 \%)$ \\
\hline Mayor o igual 20 mm & $49(14 \%)$ \\
\hline DENSIDAD & \\
\hline$<1000$ & $234(67 \%)$ \\
\hline Mayor o igual 1000 & $116(33 \%)$ \\
\hline COMPLICACIONES & $16(5 \%)$ \\
\hline Sí & $334(95 \%)$ \\
\hline No & \\
\hline
\end{tabular}

Abreviaturas: TOTAL, CAL = total de cálculos; IZQ. $C A L=$ cálculos en el lado izquierdo; DER. CAL = cálculos en el lado derecho.

En el análisis de regresión se evidenció que, controlando por litiasis bilateral, densidad y cirugías previas, los cálculos con un tamaño mayor de $20 \mathrm{~mm}$, representaron un factor de riesgo para el desarrollo de complicación infecciosa posquirúrgica (-Tabla 4 ).

\section{Discusión}

Este es el primer estudio colombiano realizado con una cohorte grande de pacientes con litiasis renal programados para procedimientos urológicos, y que describe la experiencia
Tabla 2 Longitud y densidad de los cálculos renales según la ubicación (Total de cálculos)

\begin{tabular}{|l|l|}
\hline VARIABLES & Total \\
\hline CÁLICE & \\
\hline Tamaño (mm), Promedio (D.E.) & $7,81(6,00)$ \\
\hline Densidad (UH), Promedio (D.E.) & $546,68(239,17)$ \\
\hline Anterior y posterior & $1(0,15 \%)$ \\
\hline Inferior & $255(38,17 \%)$ \\
\hline Medio & $174(26,05 \%)$ \\
\hline Medio e inferior & $42(6,29 \%)$ \\
\hline Superior & $131(19,61 \%)$ \\
\hline Superior e inferior & $12(1,80 \%)$ \\
\hline Superior y medio & $19(2,84 \%)$ \\
\hline Superior medio e inferior & $34(5,09 \%)$ \\
\hline URÉTER & \\
\hline Tamaño (mm), Promedio (D.E.) & $7,18(5,14)$ \\
\hline Densidad (UH), Promedio (D.E.) & $564,96(208,56)$ \\
\hline Distal & $106(53,27 \%)$ \\
\hline Medio & $20(10,05 \%)$ \\
\hline Proximal & $73(36,68 \%)$ \\
\hline PELVIS RENAL & \\
\hline Tamaño (mm), Promedio (D.E.) & $12,64(7,47)$ \\
\hline Densidad (UH), Promedio (D.E.) & $655,08(208,93)$ \\
\hline VEJIGA & $10,45(6,83)$ \\
\hline Tamaño (mm), Promedio (D.E.) & $415,20(193,30)$ \\
\hline Densidad (UH), Promedio (D.E.) & \\
\hline & \\
\hline
\end{tabular}

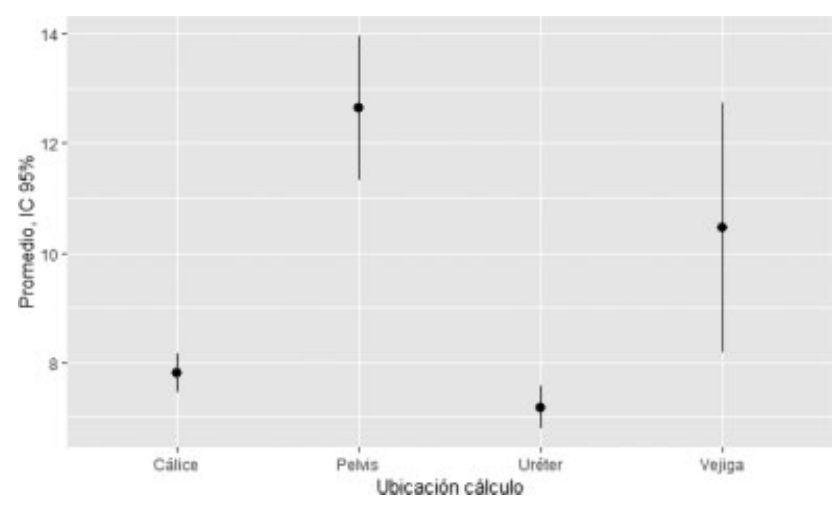

Fig. 1 Promedio del tamaño de los cálculos según ubicación anatómica.

de un centro especializado de urología. Se encontró que la tasa de complicación infecciosa fue baja. Además, se evidenció que el tamaño del cálculo fue un factor asociado al desarrollo de complicación infecciosa posoperatoria.

La incidencia de la litiasis renal en los últimos años se ha incrementado en un $40 \%{ }^{17,18}$ y se podría deber al uso inadecuado de antibióticos, y a la disbiosis que genera en el microbioma de orina. ${ }^{19}$ El problema se agrava cuando la persistencia global de la litiasis puede alcanzar hasta un 
Tabla 3 Factores clínicos asociados a complicación infecciosa en pacientes con litiasis renal

\begin{tabular}{|c|c|c|c|c|}
\hline VARIABLES & $\begin{array}{l}\text { INFECTADOS } \\
N=14\end{array}$ & $\begin{array}{l}\text { NO INFECTADOS } \\
N=336\end{array}$ & Valor $p$ & OR crudo (IC95\%) \\
\hline \multicolumn{5}{|l|}{ EDAD } \\
\hline $\begin{array}{l}\text { Mayor o igual } 45 \text { años } \\
\text { Menor a } 45 \text { años }\end{array}$ & $\begin{array}{l}10(71,43) \\
4(28,57)\end{array}$ & $\begin{array}{l}179(53,27) \\
157(46,73)\end{array}$ & 0,29 & $2,19(0,67-7,13)$ \\
\hline \multicolumn{5}{|l|}{ NÚMERO DE CÁLCULOS } \\
\hline $\begin{array}{l}\text { Mayor o igual } 3 \\
\text { Menor de } 3\end{array}$ & $\begin{array}{l}12(85,71) \\
2(14,29)\end{array}$ & $\begin{array}{l}269(80,06) \\
67(19,94)\end{array}$ & 0,86 & $1,50(0,33-6,84)$ \\
\hline \multicolumn{5}{|l|}{ IZQ. CAL (número) } \\
\hline Media (Desv. Estándar) & $1,43(1,28)$ & $1,40(1,23)$ & 0,92 & \\
\hline \multicolumn{5}{|l|}{ DER. CAL (número) } \\
\hline Media (Desv. Estándar) & $0,79(1,05)$ & $1,48(1,30)$ & 0,03 & \\
\hline \multicolumn{5}{|l|}{ TAMAÑO } \\
\hline $\begin{array}{l}\text { Mayor o igual } 6 \mathrm{~mm} \\
\text { Menor a } 6 \mathrm{~mm}\end{array}$ & $\begin{array}{l}12(85,71) \\
2(14,29)\end{array}$ & $\begin{array}{l}169(50,30) \\
167(49,70)\end{array}$ & 0,02 & $5,93(1,31-26,90)$ \\
\hline \multicolumn{5}{|l|}{ DENSIDAD } \\
\hline Media (Desv. Estándar) & $606,89(301,09)$ & $543,45(230,68)$ & 0,55 & \\
\hline \multicolumn{5}{|l|}{ CIRUGÍAS PREVIAS } \\
\hline Sí & $12(85,71)$ & $261(77,91)$ & 0,72 & $1,70(0,37-7,77)$ \\
\hline \multicolumn{5}{|l|}{ SEXO } \\
\hline $\begin{array}{l}\text { Femenino } \\
\text { Masculino }\end{array}$ & $\begin{array}{l}9(64,28) \\
5(35,71)\end{array}$ & $\begin{array}{l}192(57,14) \\
144(42,86)\end{array}$ & 0,80 & $1,35(0,44-4,11)$ \\
\hline \multicolumn{5}{|l|}{ MÚLTIPLES CÁLCULOS } \\
\hline $\begin{array}{l}\text { Sí } \\
\text { No }\end{array}$ & $\begin{array}{l}12(85,71 \%) \\
2(14,29 \%) \\
\end{array}$ & $\begin{array}{l}269(80,06 \%) \\
67(19,94 \%)\end{array}$ & 0,85 & $1,49(0,33-6,84)$ \\
\hline \multicolumn{5}{|l|}{ BILATERAL } \\
\hline $\begin{array}{l}\text { Sí } \\
\text { No }\end{array}$ & $\begin{array}{l}5(35,71 \%) \\
9(64,29 \%) \\
\end{array}$ & $\begin{array}{l}193(57,44 \%) \\
143(42,56 \%)\end{array}$ & 0,18 & $0,41(0,13-1,25)$ \\
\hline \multicolumn{5}{|l|}{ LOCALIZACIÓN } \\
\hline Cálice & $13(92,86 \%)$ & $280(83,33 \%)$ & 0,56 & $2,6(0,33-20,28)$ \\
\hline Uréter & $6(42,86 \%)$ & $179(53,27 \%)$ & 0,62 & $0,66(0,22-1,94)$ \\
\hline Pelvis renal & $1(7,14 \%)$ & $31(9,23 \%)$ & 1 & $0,76(0,09-5,98)$ \\
\hline Uretra & $0(0 \%)$ & $1(0,3 \%)$ & 1,00 & NA \\
\hline Vejiga & $1(7,14 \%)$ & $8(2,38 \%)$ & 0,81 & $3,15(0,37-27,11)$ \\
\hline \multicolumn{5}{|l|}{ TAMAÑO } \\
\hline Mayor o igual 20 mm & $5(35,71 \%)$ & $44(13,10 \%)$ & 0,04 & $3,69(1,18-11,51)$ \\
\hline \multicolumn{5}{|l|}{ DENSIDAD } \\
\hline Mayor o igual 1000 & $2(14,29 \%)$ & $114(33,93 \%)$ & 0,21 & $0,32(0,07-1,47)$ \\
\hline
\end{tabular}

Abreviaturas: IZQ. CAL = cálculos en el lado izquierdo; DER. $C A L=$ cálculos en el lado derecho.

$50 \%{ }^{20}$ Para el tratamiento de la urolitiasis, se utiliza la expulsión activa, ondas de choques, procedimientos endoscópicos y las ureterolitotomias. La indicación del tratamiento depende de la ubicación y el tamaño de los cálculos. $^{11}$

Estudios han descrito que la tasa de complicación global posterior al procedimiento urológico en pacientes con litiasis renal se encuentra entre el $7,4 \%$ y el $12 \%$, siendo la de tipo infecciosa la más frecuente. ${ }^{21,22}$ En el estudio, la tasa de complicación infecciosa fue $4,00 \%$ inferior a lo reportado en los estudios. Eso se podría deber a dos factores. El primero, es que la mayoría de los cálculos tuvieron un tamaño menor a $10 \mathrm{~mm}$; la segunda razón es que los datos se recolectaron de una institución especializada en la atención de pacientes de urología, y donde los cirujanos cuentan con amplia experiencia en el manejo quirúrgico de la urolitiasis.

Como un factor de riesgo para la complicación infecciosa posoperatoria, se encontró el hecho de tener un cálculo con una longitud mayor de $20 \mathrm{~mm}$, aspecto que ha sido descrito en otros estudios principalmente como un importante 
Tabla 4 Análisis de regresión. Factores asociados a complicación infecciosa posoperatoria

\begin{tabular}{|l|l|l|}
\hline Variable & OR Estimado & OR Ajustado \\
\hline Litiasis bilateral & $0,41(0,13-1,25)$ & $0,42(0,13-1,31)$ \\
\hline $\begin{array}{l}\text { Tamaño mayor } \\
\text { o igual } 20 \mathrm{~mm}\end{array}$ & $3,69(1,18-11,51)$ & $4,49(1,39-14,52)$ \\
\hline $\begin{array}{l}\text { Densidad mayor } \\
\text { o igual 1000 UH }\end{array}$ & $0,32(0,07-1,47)$ & $0,31(0,07-1,47)$ \\
\hline $\begin{array}{l}\text { Cirugías } \\
\text { previas (si) }\end{array}$ & $1,70(0,37-7,77)$ & $1,81(0,38-8,52)$ \\
\hline
\end{tabular}

predictor de la persistencia global de la urolitiasis, junto con la litiasis múltiple y la bilateralidad. ${ }^{20,23}$ Estudios han reportado como factores de riesgo a infección, la bacteriuria asintomática por microorganismo multirresistente, ${ }^{22,24}$ el tiempo del procedimiento y la presencia de dispositivo urinario. Por lo tanto, el tamaño del cálculo es un criterio que se debe tener en cuenta para la selección del procedimiento urológico, y se podría considerar un factor de riesgo tanto para la complicación infecciosa posoperatoria como para la litiasis recurrente.

La bilateralidad se presentó con menor frecuencia en los pacientes con complicación infecciosa un resultado inesperado, pero que ha sido reportado en otros estudios. Recientemente Rivera y col., comparó la tasa de complicación en pacientes con nefrolitotomia percutánea unilateral con bilateral, encontrando un mayor tiempo quirúrgico en la urolitiasis bilateral, pero con tasas de complicación similares. ${ }^{25}$ Se deberán realizar otros estudios que evalúen el aporte de la bilateralidad a la tasa de complicación infecciosa.

El estudio presenta algunas limitaciones que hay que tener en cuenta. En primera medida no se logró realizar un análisis estratificado del riesgo de complicación infecciosa por procedimiento urológico. Estudios han demostrado que el riesgo de infección es diferente según los procedimientos, debido al grado de manipulación de la vía urinaria y el tiempo quirúrgico. En ese mismo sentido, no se logró analizar la tasa de infección según el medio de desintegración del cálculo, sin embargo, en la institución para los cálculos a nivel renal o uréter proximal se utiliza litofragmentación laser, mientras que para los del uréter distal se realiza litofragmentación neumática balística. Otra de las limitaciones fue la no inclusión de variables como tabaquismo, característica química del cálculo, uso de antibiótico previo, utilización de catéter urinario, y duración del catéter doble J.

\section{Conclusión}

Se concluye que la tasa de complicación infecciosa posoperatoria en pacientes con urolitiasis y programados para procedimientos urológicos es baja. Una longitud del cálculo mayor de $20 \mathrm{~mm}$ es un factor asociado a la infección posoperatoria.
Conflicto de intereses:

Los autores declaramos no tener ningún conflicto de intereses.

\section{Agradecimientos}

Los autores agradecen al Centro Especializado de Urología de la ciudad de Neiva, Colombia.

\section{Referencias}

1 Gottlieb M, Long B, Koyfman A. The evaluation and management of urolithiasis in the ED: A review of the literature. Am J Emerg Med 2018;36(04):699-706

2 Ingimarsson JP, Krambeck AE, Pais VM Jr. Diagnosis and Management of Nephrolithiasis. Surg Clin North Am 2016;96 (03):517-532

3 Moe OW. Kidney stones: pathophysiology and medical management. Lancet 2006;367(9507):333-344

4 Portis AJ, Sundaram CP. Diagnosis and initial management of kidney stones. Am Fam Physician 2001;63(07):1329-1338

5 Jiao B, Lai S, Xu X, Zhang M, DiaoT, Zhang G. The efficacy of flexible ureteroscopy lithotripsy and miniaturized percutaneous nephrolithotomy for the treatment of renal and proximal ureteral calculi of $\leq 2 \mathrm{~cm}$ : A retrospective study. Medicine (Baltimore) 2019;98(11):e14535

6 Sorokin I, Mamoulakis C, Miyazawa K, Rodgers A, Talati J, Lotan Y. Epidemiology of stone disease across the world. World J Urol 2017;35(09):1301-1320

7 Adela-Emilia GAyala. Litiasis Renal. Etiopatogenia, clínica y factores de riesgo. Rev Clínica Med Fam 2008;2:32-38

8 Kravdal G, Helgø D, Moe MK. Kidney stone compositions and frequencies in a Norwegian population. Scand J Urol 2019;53(23):139-144

9 Taylor EN, Stampfer MJ, Curhan GC. Dietary factors and the risk of incident kidney stones in men: new insights after 14 years of follow-up. J Am Soc Nephrol 2004;15(12):3225-3232

10 Fernández Alcalde ÁA, Ruiz Hernández M, Gómez Dos Santos V, et al. Comparación entre nefrolitotomía percutánea y ureteroscopia flexible para el tratamiento de litiasis renales de entre 2 y 3cm. Actas Urol Esp 2019;43(03):111-117

11 Türk C, Knoll T, Petrik A, Sarica K, Straub M, Seitz C. Guidelines on urolithiasis. Eur Assoc Urol 2017

12 Pan J, Chen $\mathrm{Q}$ Xue W, et al. RIRS versus MPCNL for single renal stone of $2-3 \mathrm{~cm}$ : clinical outcome and cost-effective analysis in Chinese medical setting. Urolithiasis 2013;41(01):73-78

13 Zengin K, Tanik S, Karakoyunlu N, et al. Retrograde intrarenal surgery versus percutaneous lithotripsy to treat renal stones 2$3 \mathrm{~cm}$ in diameter. BioMed Res Int 2015;2015:914231

14 Pieras E, Tubau V, Brugarolas X, Ferrutxe J, Pizá P. Comparative analysis between percutaneous nephrolithotomy and flexible ureteroscopy in kidney stones of $2-3 \mathrm{~cm}$. Actas Urol Esp Engl Ed 2017;41:194-199

15 Ramos-Castaneda JA, Ruano-Ravina A, Munoz-Price LS, et al. Risk of infection in patients undergoing urologic surgery based on the presence of asymptomatic bacteriuria: A prospective study. Am J Infect Control 2019;47(12):1474-1478

16 Team Rs. RStudio: integrated development for R. RStudio IncBoston MA URL Httpwww Rstudio Com 2015:42

17 Stamatelou KK, Francis ME, Jones CA, Nyberg LM Jr, Curhan GC. Time trends in reported prevalence of kidney stones in the United States: 1976-1994. Kidney Int 2003;63(05):1817-1823

18 Hesse A, Brändle E, Wilbert D, Köhrmann K-U, Alken P. Study on the prevalence and incidence of urolithiasis in Germany comparing the years 1979 vs. 2000. Eur Urol 2003;44(06): 709-713 
19 Tasian GE, Jemielita T, Goldfarb DS, et al. Oral Antibiotic Exposure and Kidney Stone Disease. J Am Soc Nephrol 2018;29(06): $1731-1740$

20 Pieras E, Ruiz J, Vicens A, Frontera G, Grases F, Pizá P. Multivariate analysis of predictive factors in the evolution of renal lithiasis. Actas Urol Esp Engl Ed 2012;36:346-351

21 Bai T, Yu X, Qin C, et al. Identification of Factors Associated with Postoperative Urosepsis after Ureteroscopy with Holmium: Yttrium-Aluminum-Garnet Laser Lithotripsy. Urol Int 2019;103 (03):311-317

22 Ramos-Castaneda JA, Ruano-Ravina A, Salinas DF, OsorioManrique J, Segura-Cardona AM, Lemos-Luengas EV. Factors associated with multidrug-resistant bacteria in a cohort of patients with asymptomatic bacteriuria who underwent urological surgery. Am J Infect Control 2019;47(12): $1479-1483$

23 Ziemba JB, Matlaga BR. Epidemiology and economics of nephrolithiasis. Investig Clin Urol 2017;58(05):299-306

24 Ramos JA, Salinas DF, Osorio J, Ruano-Ravina A. Antibiotic prophylaxis and its appropriate timing for urological surgical procedures in patients with asymptomatic bacteriuria: A systematic review. Arab J Urol 2016;14(03):234-239

25 Rivera ME, Bhojani N, Heinsimer K, et al. A survey regarding preference in the management of bilateral stone disease and a comparison of Clavien complication rates in bilateral vs unilateral percutaneous nephrolithotomy. Urology 2018;111:48-53 